\section{The upper hand}

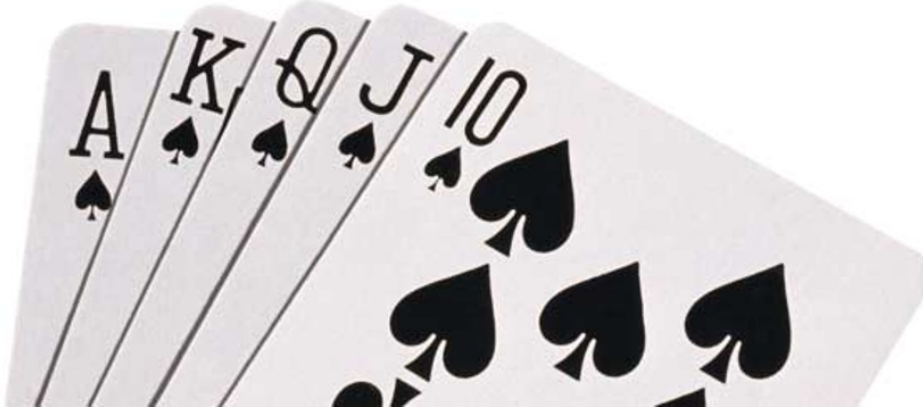

Genetic and epigenetic alterations - two equal but alternative forces in tumorigenesis? Perhaps not, as a recent study by Manuel Perucho and colleagues in Cancer Cell indicates that genetics might still have the upper hand.

The hypothesis that a mutator phenotype can contribute to tumorigenesis is supported by the fact that loss of mismatch repair (MMR) proteins leads to microsatellite instability (MSI) and colorectal cancer. But loss of MMR can also be caused by epigenetic silencing of the MMR gene $M L H 1$, which could occur because of a CpG island methylator phenotype (CIMP). The authors therefore sought to identify whether CIMP precedes MSI and the mutator phenotype, and whether it has a significant role in colorectal cancer.

They began by performing methylation-specific PCR amplification of six gene promoters - all of which are known to be hypermethylated in colorectal cancer - in a panel of 207 colorectal cancers. Interestingly, the number of methylated loci per tumour form a gradual pattern, rather than the bi-modal distribution that would be expected if the CIMP phenotype occurred at a specific stage in tumour progression and was then instrumental in causing a significant increase in methylation. However, it was possible that these few loci were not sufficient to detect the phenotype, so a global analysis of methylation content was performed by methylation-sensitive amplified fragment length polymorphism. This increase in the number of loci examined - up to 35 confirmed that the frequency of methylated loci still reflects a normal distribution, characteristic of random events, whereas the frequency of microsatellite mutations reflects a bi-modal distribution.

So, it seems that the mutator phenotype is not caused by a methylator phenotype. To investigate this further, the authors compared features of colorectal tumours either with or without a methylator phenotype (at least two methylated promoters out of the original six) and a mutator phenotype (positive for MSI). Whereas MSI-positive and MSI-negative tumours had several notable genotypic and phenotypic differences, there were only two significant differences between tumours that did and did not possess the methylator phenotype - methylator-positive tumours were generally found in the proximal colon and occurred in older patients. However, these two features precede transformation.

The mutator phenotype therefore seems to be dominant over the methylator phenotype in colorectal cancer, and although methylation of MLH1 can induce MSI, it seems that acquisition of the mutator phenotype is still the important event. Whether this is also the case in other tumour types for which CIMP has been proposed to have a role remains to be determined.

Emma Greenwood

(2) References and links ORIGINAL RESEARCH PAPER Yamashita, K. et al. Genetics supersedes epigenetics in colon cancer phenotype. Cancer Cell 4, 121-131 (2003) WEB SITE

Manuel Perucho's lab: http://www.burnhaminst.org/FacultyAndResearch/faculty/manuel_peru cho_bio.asp

\section{TRIAL WATCH}

\section{Tobacco and DNA damage}

Although $80-90 \%$ of patients with lung cancer smoke, the percentage of all smokers who develop lung cancer is low. This indicates that risk factors other than the carcinogenic effect of tobacco smoke are also important. Tamar Paz-Elizur and colleagues have carried out a case-control study to investigate whether reduced repair of oxidative DNA damage — known to be caused by tobacco carcinogens - is associated with lung cancer.

The study included 68 patients with non-small-cell lung cancer (NSCLC) and 68 matched healthy control participants. The study included people who had never smoked or who currently smoked, but not those who had smoked previously. Blood samples were taken from all participants - for the patients this was between 4 months before surgery to up to 1 year after surgery - and peripheral-blood mononuclear cells (PBMCs) were isolated. Non-tumour lung samples were also collected from patients with NSCLC during surgery.

The authors focused on the repair of DNA containing 8 -oxoguanine, a lesion formed by tobacco smoke. This adduct is removed from DNA by base-excision repair, initiated by the enzyme 8-oxoguanine DNA $N$-glycosylase (OGG). The authors used a radiolabelled DNA oligonucleotide containing a single 8-oxoguanine residue as a substrate to assess OGG activity in the PBMC samples. If an active enzyme removed the lesion, the resulting DNA fragments could be seen on a polyacrylamide gel.

The mean OGG activity in the NSCLC patients was $5.8 \mathrm{U} / \mu \mathrm{g}$ protein, which was significantly lower than the activity seen in the control participants (7.1 U/Mg protein). $41 \%$ of NSCLC patients but only $4 \%$ of control participants had OGG activity values less than or equal to 5.5. An analysis of OGG activity in lung samples from seven NSCLC patients showed a good correlation with the OGG activity in the PBMC samples taken from the same patients, indicating that activity in PBMC is a good surrogate marker for activity in the lungs. Blood samples that were taken from NSCLC patients up to 4 months after surgery also showed lower OGG activity than the control participants, indicating that OGG activity is not related to the presence of the lung tumour.

After adjustment for age and smoking status, individuals with the lowest tertile of OGG activity had an increased risk of NSCLC compared with individuals in the highest tertile (adds ratio 4.8). Smoking status made no difference to this risk. The relative risk for smokers was 34-fold higher in those with $6.0 \mathrm{U} / \mu \mathrm{g}$ protein or 124 -fold higher in those with $4.0 \mathrm{U} / \mu \mathrm{g}$ protein than for nonsmokers with a normal OGG activity of $7.0 \mathrm{U} / \mu \mathrm{g}$ protein.

This assay might be a valuable addition to assessing risk of developing NSCLC in smokers, and smoking cessation might be a particularly effective prevention strategy in individuals with reduced OGG activity.

ORIGINAL PAPER Paz-Elizur, T. et al. DNA repair activity for oxidative damage and risk of lung cancer. J. Natl Cancer Inst. 95, 1312-1319 (2003)

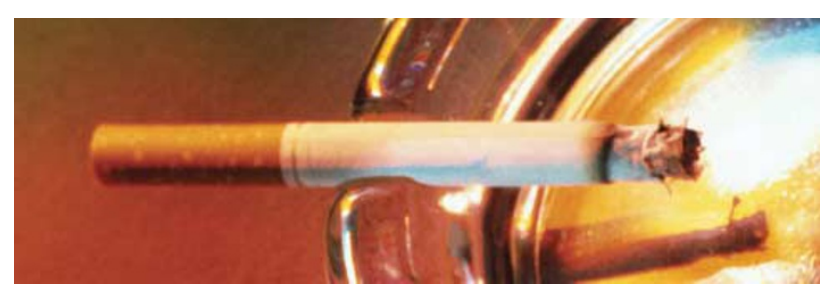

